\title{
Sport Nutrition in Childhood: Meeting the Metabolic Demands of Growth and Exercise
}

\author{
C.L. Zanker \\ Carnegie Faculty of Sport and Education, Leeds Metropolitan University, Leeds, UK
}

\section{Key Words}

Exercise, nutrition - Growth/puberty, sport nutrition •

Physical performance $\cdot$ Fuel metabolism

\begin{abstract}
The primary goals of childhood are optimal growth and maturation. These developmental processes demand positive energy and nitrogen balance. Regular physical activity can enhance childhood development; however, strenuous physical training may reduce the body's stores of energy and nitrogen, both of which must be replaced through the diet. Physically active adolescents are susceptible to disordered eating, which can lead to protein-energy malnutrition. The consequences of negative energy and nitrogen balance include delayed, retarded or arrested growth and pubertal maturation. Currently, there is scant evidence to suggest that physical training has a direct negative impact on growth and maturation. A well-balanced diet is essential for the maintenance of physical performance and the preservation of health in all physically active individuals, regardless of age. Food provides the chemical elements required for the production of energy by exercising skeletal muscle, the post-exercise replenishment of fuel stores and the regeneration of damaged muscle. The dietary requirements of physically active children alter with increasing body size and pubertal
\end{abstract}

progression. The maturation state is an important determinant of the metabolic and physiological responses to exercise, and so influences both physical performance capacity and dietary needs. Studies of fuel metabolism in children suggest that they are well equipped for sustained aerobic activity, but that their capacity for anaerobic performance is limited by their maturation state. Importantly, ethical restrictions preclude the type of detailed and invasive research that has identified the nutritional requirements of physically active adults. Nevertheless, it would seem that like such adults, physically active children have greater dietary demands for energy, carbohydrate, protein and water than their sedentary counterparts. This article justifies the nutritional requirements of athletic children on the basis of the physiological and metabolic demands of survival, growth and physical activity.

Copyright $\odot 2006$ Nestec Ltd., Vevey/S. Karger AG, Basel

\section{Introduction}

Childhood represents a phase of significant growth and physical maturation. These processes depend on regular physical activity and sound nutrition. It is also during childhood that personality develops and talents emerge. Such talents include athletic ability and proficiency in var-

\section{KARGER}

Fax +41613061234

E-Mail karger@karger.ch

www.karger.com
(C) 2006 Nestec Ltd., Vevey/S. Karger AG, Basel

$0517-8606 / 06 / 0642-0063 \$ 23.50 / 0$

Accessible online at:

www.karger.com/ane
Dr. Cathy L. Zanker

Carnegie Research Institute

Carnegie Faculty of Sport and Education, Leeds Metropolitan University

Headingley Campus, Leeds LS6 3QS (UK)

Tel. +44 113283 2600, Fax +44 113283 7575, E-Mail c.zanker@leedsmet.ac.uk 
ious sports. Carefully structured physical training in conjunction with a well-balanced diet can promote athletic performance in childhood and prepare the body for future competition in sport.

Prior to puberty, the physical performance capacity of boys and girls is quite comparable. However, with the progression of puberty, a significant divergence between the sexes becomes apparent $[1,2]$. In both boys and girls, physical training augments normal pubertal increments in muscle size, strength, aerobic and anaerobic power, all of which are important determinants of physical performance. Nevertheless, in girls, the development of these physiological and performance features through adolescence is much less prominent than in boys, and significant accumulations of body fat in girls may hinder athletic ability $[1,3]$. Strenuous training and low body weight have been associated with neuroendocrine dysfunction, gonadal suppression and a multitude of endocrine disturbances that disrupt the normal balance of bone turnover $[4,5]$. The consequences of these disturbances include stunted growth, retarded or arrested puberty and inadequate bone formation, all of which may impair existing and future health $[1,3]$.

Importantly, there is convincing evidence that endocrine disturbances and imbalanced bone metabolism in athletic children are largely attributable to primary nutritional insufficiency that is exacerbated by the added energy cost of physical training, rather than to training itself $[6,7]$. On the basis of this evidence, it is essential that children engaging in strenuous sports consume a diet that adequately satisfies the nutritional demands of both their physical training and normal growth. This article justifies the nutritional requirements of athletic children on the basis of the physiological and metabolic demands of survival, growth and physical activity. The effects of growth and maturation on the metabolic and physiological responses to exercise are summarized, with discussion of the cooperative impact of such factors on physical performance and dietary needs.

\section{Ethical Issues in Relation to Research in Athletic Children}

When considering the nutritional requirements of athletic children, it should be acknowledged that the evidence base that guides such requirements is limited. This contrasts with extensive research evidence that addresses the principal nutritional features associated with exercise in adults. This evidence has been used to develop dietary guidelines for men and women training for and competing in a variety of sports [8]. The relative lack of comparable research evidence in athletic children is primarily attributable to ethical and methodological restrictions on the type of data collection, which have increased over recent years $[9,10]$. Techniques that have been applied in metabolic studies of exercising adults, although informative, frequently exceed the boundaries of ethical acceptability for use in children.

In adults, measurement of blood-borne and intramuscular fuels or their metabolites has furthered understanding of the rates of turnover of energy substrates during exercise. In addition, the use of stable isotopes has allowed investigation of the kinetics of substrate mobilization and utilization. These procedures are highly informative and instrumental to the detailed study of energy balance and fuel metabolism during exercise. However, they are accompanied by a degree of risk, discomfort or pain that is deemed excessive, or difficult to quantify in children. Consequently, research involving invasive procedures is generally permitted only for use in sick children undergoing such procedures as part of their healthcare $[9,10]$. When studying various aspects of metabolism in healthy children, international ethical regulations for biomedical research in humans [11] emphasize the use of indirect techniques which are noninvasive and nonintrusive to a child's lifestyle. These regulations clearly limit the depth and precision of research that may be conducted in children, but importantly they protect the child's dignity, health and welfare.

\section{Establishing Nutritional Guidelines for Physically Active Individuals}

The primary determinants of the nutritional requirements of exercise concern the 'extra' energy demands of an activity beyond that of sedentary demands and the absolute consumption of different metabolic fuels $[12,13]$. Hydration also exerts a significant influence on physical performance and health, and fluid intake from food and beverages should balance fluid losses through excretion and perspiration. Research in adults has addressed a variety of exercise-related nutritional issues, many of which are summarized in the Joint Position Statement on Nutrition and Athletic Performance of the American College of Sports Medicine (ACSM), the American Dietetic Association (ADA) and the Dietitians of Canada (DC) [8]. These nutritional issues include: 
(1) The energy demands and specific metabolic costs of a variety of sports and activities [14, 15].

(2) The regulation of fuel metabolism in exercising muscle performed at different exercise intensities and over varying durations $[16,17]$.

(3) Sex differences in fuel metabolism during exercise $[18,19]$.

(4) The role of diet manipulation, including the timing of meal ingestion in relation to exercise and the use of dietary supplements to modulate physical performance and/or to enhance post-exercise recovery [20,21].

Established dietary requirements for physically active adults focus on the optimization of performance and the maintenance of good health [8]. However, much of this knowledge is absent in children and the extent to which findings from adult studies apply to children is presently unclear. The fundamental nutritional concern for athletic children is the provision of adequate energy and specific nutrients for normal growth and maturation; the ergogenic (i.e. performance-enhancing) potential of food is of secondary importance $[3,22]$. Moreover, the physiology of children is less advanced than that of adults. This is especially true of prepubertal children who, when compared to adults, exhibit dissimilar responses to exercise in terms of energy expenditure for a given activity, patterns of fuel metabolism and thermoregulation [2, 22, 23]. Nevertheless, there is evidence to suggest that the metabolic and physiological responses of older adolescents to various activities are comparable to those of adults of the same sex $[2,22,23]$.

Current knowledge of the special dietary needs of athletic children of different chronological or biological age is quite limited. Consequently, nutritional guidelines for child athletes are quite vague and are based upon the following three main factors:

(1) An established knowledge of the nutritional demands of childhood growth and maturation.

(2) The physiology and physical performance potential of children of a given chronological or biological age.

(3) Adaptations from adult data obtained from metabolic investigations conducted in association with exercise and physical training.

Ultimately, vital indicators for the nutritional adequacy of the diet of a child athlete are the child's state of general health and well-being, their rate of linear growth and maturation, and the appropriateness of their weight gain over a specified period of time $[1,7,22]$. For child athletes, diet may be considered to satisfy the nutritional demands of training and normal growth when, over a period of months, the child continues to gain in stature and weight in tempo with the normal percentiles on a validated growth chart, and there is no delay or deterioration in pubertal development $[1,7]$. The presence of chronic energy or protein deprivation in children is indicated by inadequate gains in height and weight that deviate from validated growth curves, or a deficit in weight for height, as indicated through the assessment of body mass index. Behavioral changes, including fatigue, lethargy, irritability and apathy, or physical malfunction, such as frequent infection and soft tissue injury, may also signify nutritional insufficiency, including dehydration, in child athletes [22, 24].

\section{Respiratory Pathways in Exercising Skeletal Muscle}

Energy for muscle activity is obtained from the catabolism of adenosine triphosphate (ATP). The quantity of ATP within muscle cells is limited and sufficient to power muscle activity for only a few seconds. To sustain exercise, ATP must therefore be resynthesized as rapidly as it is utilized $[12,13]$. During exercise, the activity of the various respiratory pathways for ATP synthesis within the recruited muscles determines the rate and extent of catabolism of stored and circulating fuels, and thereby influences the nutritional demands of exercise $[12,13]$. In adults and children there are three primary respiratory pathways operative within exercising skeletal muscle:

(1) Creatine phosphokinase reaction involves the phosphorylation of adenosine diphosphate via the anaerobic catabolism of creatine phosphate (PCr). It provides energy for short bursts $(<10 \mathrm{~s})$ of maximal intensity muscle activity and is a short-lived energy system owing to the limited quantities of stored PCr.

(2) Anaerobic glycolysis involves the anaerobic catabolism of muscle glycogen to lactic acid. It provides energy for brief $(<1 \mathrm{~min})$ maximal intensity exercise and is a short-lived energy system because the major byproduct (lactic acid), amongst other metabolic wastes, interferes with excitation-contraction coupling in skeletal muscle.

(3) Aerobic respiration involves the complete catabolism of fuels (mainly glucose and fatty acids) via the tricarboxylic acid cycle and oxidative phosphorylation to carbon dioxide and water. It provides energy over a sustained duration because there is no accumulation of toxic metabolites.

In both adults and children, the main factor that determines the operative respiratory pathways during exercise is the intensity of muscle activity, and hence the rate of degradation of ATP $[12,16]$. Exercise duration is also in- 
fluential because, as muscle activity continues, intramuscular and hepatic glycogen stores are selectively depleted and alternative fuels are increasingly called upon [17, 20]. Fatigue develops when the force of muscular contraction declines and a given level of exercise intensity is unsustainable. Exhaustion denotes a point at which it is no longer possible to continue exercise [25]. Fatigue and exhaustion are normally linked to a depletion of energy substrate, an accumulation of metabolic wastes, dehydration and/or hyperthermia [25].

\section{Fuels for Energy Production}

In accordance with the operation of the aforementioned respiratory pathways, the primary diet-derived fuels for ATP synthesis within the exercising muscles of both adults and children are the muscles' own stores of PCr, glycogen and triglyceride, together with blood-borne glucose and non-esterified fatty acids [16, 17]. During sustained exercise, the body's limiting energy sources are glycogen and glucose derived from dietary carbohydrates $[17,20]$. The rate and extent of catabolism of these fuels are governed by exercise intensity and duration $[26,27]$. Despite being as energy-rich as glucose per unit mass, amino acids contribute only a minor proportion to ATP synthesis during exercise in healthy, well-nourished individuals, except for exercise that is both prolonged (e.g. $>90 \mathrm{~min}$ ) and intense (e.g. $>75 \%$ maximal aerobic power or $\left.\dot{\mathrm{V}}_{2 \max }\right)[17,20]$.

Prolonged, strenuous exercise may ultimately deplete muscle and liver glycogen stores because ATP must be synthesized at a high rate over a sustained duration [20, 27]. Muscle glycogen and blood glucose contribute significantly to such exercise. As glycogen stores dwindle with continued exercise, and hypoglycemia ensues, a fall in the plasma concentration ratio of insulin/glucagon and an increase in plasma cortisol concentration stimulate muscle proteolysis $[21,28]$. Such proteolysis leads to the release of free amino acids. Active muscles are able to extract and oxidize increasing quantities of the branched-chain amino acids, leucine, isoleucine and valine, whilst other amino acids may be transaminated to produce alanine and glutamine for hepatic gluconeogenesis $[28,29]$. However, amino acids are not 'ideal' fuels for ATP synthesis because enhanced catabolism of tissue protein poses the risk of negative nitrogen balance. When sustained, negative nitrogen balance impairs health and may result in immune suppression, soft tissue injury and impaired bone formation $[4,21]$. Because of the impor- tance of positive nitrogen balance for growth, it is likely that the deleterious health consequences of exercise-induced glycogen depletion are greater in children than in adults.

Lipid mobilization and fatty acid oxidation also increase with exercise duration, both in children and adults $[26,30]$. This increase in fat metabolism is elicited by a multitude of endocrine responses, which include increases in the plasma levels of cortisol, catecholamines and growth hormone, and a decrease in plasma insulin concentration [31, 32]. Interestingly, studies using indirect calorimetry to assess the non-protein respiratory exchange ratio $\left(\mathrm{VO}_{2} / \dot{\mathrm{VCO}}_{2}\right)$ indicate that the contribution of fatty acid oxidation to energy production is greater in children than adults exercising at the same relative exercise intensity (where the latter is expressed as $\% \mathrm{~V}_{2 \max }$ ) [33-35]. This trend seems to diminish during adolescence, especially in boys $[23,36]$. This implicates a role for sex hormones, in particular androgens, in the regulation of fuel metabolism. The nutritional implications of a greater capacity for fatty acid oxidation in prepubertal children include the conservation of glycogen and blood glucose, which may reduce the requirement for dietary carbohydrate.

\section{Physical and Physiological Determinants of Physical Performance}

Excellence in sports performance depends upon inherent physical and physiological characteristics ('good genes'), well-structured physical training to promote innate characteristics, and sound nutrition to facilitate optimal adaptation to training [37]. The primary physical and physiological determinants of sports performance that are influenced by nutrition are strength, speed, power and endurance. The relative importance of these characteristics to sports performance varies between different sports and it is unusual that they are all critical attributes for proficiency in a given sport. These physical and physiological characteristics are defined as follows [37].

Strength denotes the ability to generate force and is governed by the size of individual muscles and agonist muscles, coupled to the coordination of these muscles' motor units. Strength is a key determinant of power, which equates to the product of muscle force and the speed of contraction. Sports that demand supreme strength and power include sprinting (running, swimming or cycling), jumping, throwing and weight lifting. Gymnastics, wrestling, racquet sports and many team sports also incorpo- 
Fig. 1. Height, weight and regional areal bone mineral density (BMD) of male and female child gymnasts (age 6-8 years) expressed as standardized $(\mathrm{Z})$ scores in relation to mean values for untrained (control) children of the same sex and height. $\square=$ Girls; $\square$ = boys. Both groups of gymnasts were small and light for their age, but displayed trends towards a higher BMD than controls. Female gymnasts trained 8-10 h/ week and had trained regularly for 3-4 years. Male gymnasts trained 4-6 h/week and had trained for 1-2 years. Significance, gymnasts vs. controls of the same sex: ${ }^{*} \mathrm{p}<$ $0.05 ;{ }^{* *} \mathrm{p}<0.01$. From Zanker et al. [39].

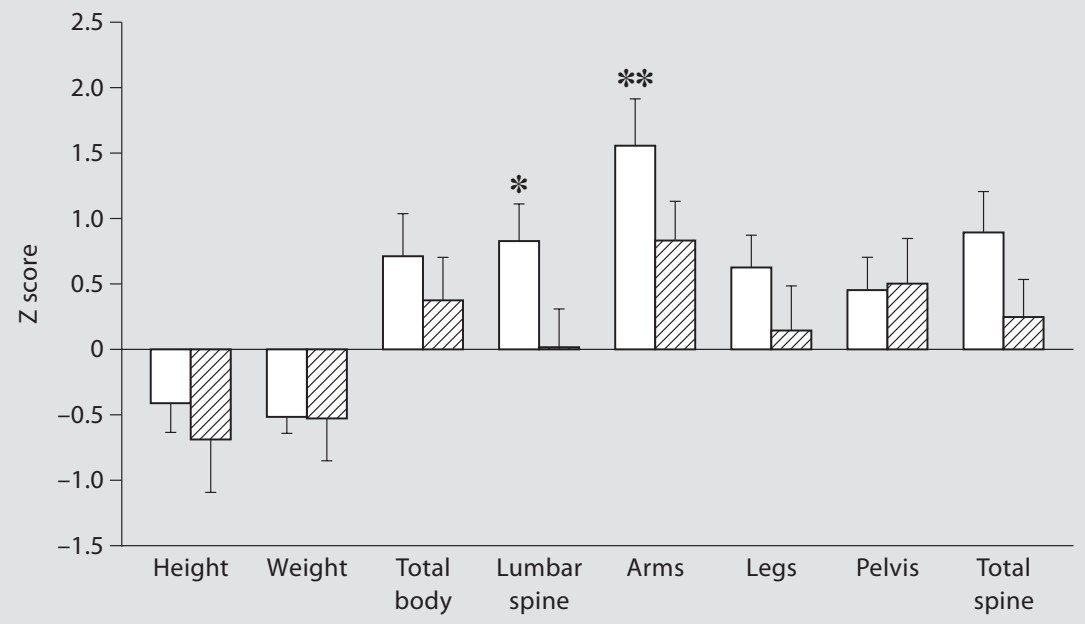

rate powerful maneuvers. Performance excellence in most sports depends upon a high ratio of strength (or power) to body mass. This is particularly pertinent to sports that involve movement of the body against the force due to gravity (e.g. running, jumping, gymnastics, etc.). In contrast, in sports such as swimming and cycling, wherein body mass is supported, absolute strength and power are of greater relevance to sports performance. Muscle activity during the performance of these sports is sustained primarily by the energy generated via the anaerobic catabolism of muscle stores of creatine phosphate and glycogen. These anaerobic respiratory systems enable a substantially greater rate of ATP synthesis than may be generated through aerobic respiration.

Endurance describes the capacity to continue exercise without fatiguing and is largely determined by cardiorespiratory fitness, together with the capacity of exercising muscle to extract and utilize oxygen in aerobic respiration. Examples of endurance sports are running, swimming and cycling over long distances, rowing and crosscountry skiing. In addition to a requirement for strength and power, racquet and team sports also demand a certain degree of cardiorespiratory and muscular endurance. Endurance sports, alongside the training for these sports, incur the highest energy cost of all physical activities. Even though the rate of energy expenditure is less for endurance sports than for strength and power sports, the total amount of energy expended during endurance activity is greater because of the longer duration for which it is sus- tained. Endurance activities are sustained predominantly via aerobic respiration, which uses a variety of sources of metabolic fuel, as governed by exercise intensity and duration, together with the magnitude of the active muscles' glycogen stores.

\section{The Development of Athletic Proficiency with Growth and Maturation}

When exploring the dietary needs of child athletes it is necessary to consider those factors that influence a child's individual requirements for energy and specific nutrients. In growing children, such factors include general health, physical size, body dimensions, maturation state and physical activity $[1,22,23]$. The latter engenders specific nutritional demands, which relate to the provision of fuel for muscle activity and the promotion of an anabolic environment to facilitate post-exercise recovery. Body size and maturation state are fundamental determinants of physical performance capacity in children, and through the course of childhood development, particularly during puberty, increases in muscle strength and aerobic power promote athletic proficiency $[1,22,23]$.

In younger children ( $<8$ years) spontaneous physical activity behaviors augment growth-related enhancements in physical performance. Running, jumping and acrobatic maneuvers are natural features of childhood play, which improve coordination, motor skills, muscle strength and 
aerobic power [38]. Moreover, in the presence of adequate nutrition, these activities appear to enhance the prepubertal acquisition of muscle and bone mass [39]. Figure 1 illustrates differences in bone density between 7 - to 8-yearold child gymnasts and normally active children and demonstrates the apparent positive effects of weight-bearing and high-impact activities on the regional accretion of bone mineral. During this phase of early development, intensive training is exceptional and generally ill-advised because of the susceptibility of the growing musculoskeletal system to overuse injuries [2]. However, for sports such as gymnastics and ballet, controlled training is customary and essential for the promotion and maintenance of flexibility, which would otherwise deteriorate with growth $[2,40]$.

With increasing age and maturity, children are able to withstand harder and more structured physical training, particularly after the onset of puberty. Prior to puberty, regardless of nutrition, skeletal muscle lacks the capability for significant hypertrophy, although neurally mediated strength gains are possible $[1,23]$. It is not until the onset of puberty that surges in the circulating levels of sex hormones, growth hormone and the insulin-like growth factors enable hypertrophic responses of skeletal muscle to activities demanding strength and power. Muscle strength per unit cross-sectional area is comparable in children, adolescents and young adults of both sexes; it is the endocrine environment at different stages of maturity in males and females that determines the capacity for exercise-induced increases in muscle bulk $[1,23]$. Sex hormones, particularly androgens, may also promote anaerobic glycolysis in skeletal muscle because anaerobic capacity and lactate production are substantially greater in men than in prepubertal boys, girls or women $[23,36]$. It is suggested that androgens induce the enzymes controlling anaerobic glycolysis and enhance the capacity for glycogen storage $[23,36]$.

$\dot{\mathrm{V}}{ }_{2 \max }$ is independent of the maturation state when expressed in relation to body mass, but is greater in larger individuals (e.g. adults versus children) when expressed in absolute terms $[23,41]$. This is because a major determinant of $\mathrm{VO}_{2 \max }$ is maximum cardiac output, and maximum stroke volume is related to heart size in healthy individuals. $\dot{\mathrm{VO}}_{2 \max }$ also depends upon the capacity of a large mass of exercising muscle to extract blood-borne oxygen and to utilize this oxygen in aerobic respiration. Adequate pulmonary function and the blood hemoglobin concentration inevitably influence oxygen transport capacity $[42,43]$. When compared to adults and older adolescents, younger children demonstrate lower mechanical efficiency and economy of oxygen utilization during walking and running $[2,22]$. This is exemplified through measurements of submaximal oxygen consumption $\left(\mathrm{V}_{2}\right)$, expressed per unit body mass, which are higher in children than adults performing a given activity.

It has been suggested that a lower economy of oxygen utilization in younger children is related to a less effective coordination of agonist-antagonist muscle groups, which leads to a 'waste' of energy [2, 22]. Given that $\mathrm{VO}_{2}$ is proportional to energy expenditure during submaximal steady-state exercise, tables listing the energy demands of various physical activities compiled from research in adults [14] usually underestimate the energy expenditures of children undertaking the same activities, even when scaled to account for differences in body size and mass. It has been estimated that in prepubertal children and adolescents, energy expenditure for a given activity exceeds adult values by $20-25$ and $10-15 \%$, respectively $[2,22]$.

The main conclusion that may be drawn in regard to the effects of physical training in prepubertal children is that specific training can enhance the development of motor skills, muscle strength and aerobic power. However, in contrast to adolescents and adults, the potential for younger children to enhance aerobic and anaerobic power through specific training is comparatively limited [2, 22]. Modulation of dietary intake (e.g. an elevation in protein intake beyond that required for the maintenance of nitrogen balance) cannot enhance these performance features before puberty $[22,24]$. During puberty, increases in whole body strength, muscular power and endurance are of substantially greater magnitude in boys than girls, both when expressed in absolute terms and in relation to body mass $[2,22]$. These sex differences are explained by differences in body size, body composition and metabolism between adolescents and adults. Whereas androgens (particularly testosterone) in adolescent boys stimulate growth of the whole body and increase lean tissue mass, estrogens in adolescent girls increase the deposition of body fat and the ratio of fat/lean tissue. Moreover, the anabolic effects of estrogens are significantly less than those of androgens $[2,22]$. Sex differences in the extent of development of physical performance attributes during adolescence account for the well-established differences in the sports performance potential of adult men and women [2, 43]. 


\section{The Role of Food and Factors That Influence the Basic Nutritional Requirements of Adults and Children}

Food provides energy substrate that is obligatory for metabolism and the maintenance of homeostasis. Energy balance, which represents the difference between energy intake and energy expenditure, is fundamental to health and physical performance. In adults, dietary energy requirements reflect the energy costs of basal metabolism, the thermic effect of food, thermoregulation and physical activity $[8,13]$. The latter includes the routine activities of day-to-day living, as well as structured sport and exercise. Physical activity has a profound affect on the rate of energy expenditure, and exercise that involves the recruitment of a large muscle mass greatly elevates the resting metabolic rate (RMR). For example, in adults during sustained whole body aerobic activity ( $>5 \mathrm{~min}$ ), RMR may increase 10 - to 20 -fold, whereas during short-lived $(<1 \mathrm{~min})$ maximal intensity anaerobic activity, a momentary 40- to 50-fold elevation in RMR is possible [13]. Consequently, repeated strenuous exercise for the purpose of physical training has the potential to elicit an energy deficit unless balanced by an adequate dietary energy intake.

In children, energy is required for the same functions as in adults; however, an extra amount beyond the demands of RMR is required to support growth and maturation. Growth depends upon the net synthesis and deposition of tissues and is therefore an anabolic process that incorporates chemical energy from food into the structural components of organs. Because the rate of tissue turnover is substantially higher in children than adults, energy expenditure per unit body mass is also higher in children [22,44]. Moreover, to sustain normal growth and maturation, children should maintain positive nitrogen balance. The latter requires nitrogen intake (from protein foods) to exceed nitrogen utilization (in synthesis and energy production). Negative energy balance inevitably leads to negative nitrogen balance because protein is catabolized to generate amino acids for use as metabolic fuel $[21,45]$. The consequence of such catabolism is weight loss, comprising both lean and fat tissue, in adults and children alike. However, in children with persistent energy deficit and negative nitrogen balance, there may be an accompanying deceleration of growth that results in short stature for chronological age. Such 'nutritional dwarfing' is characterized by a decline in the rate of linear growth with inadequate weight gain and delayed, retarded, or even arrested puberty $[7,40]$.
Nutritional insufficiency during the adolescent years, particularly of energy and protein, creates a catabolic environment that hinders the anabolic events required for normal growth and maturation. During the pubertal growth spurt, dietary energy and protein demands attain a peak to ensure positive nitrogen balance [22, 24]. Throughout this phase of development, particularly in adolescent girls, the hypothalamus is especially sensitive to acute or chronic energy deficit, hypoglycemia and lowered fat stores. These nutritional insults disturb normal diurnal variations in the circulating levels of insulin, 3,5,3'-triiodothyronine, cortisol and leptin $[4,46]$. The latter is thought to inform the hypothalamus of energy availability and to indirectly regulate pituitary function alongside the activities of peripheral endocrine glands [4, 47]. The damaging effects of energy and protein deprivation on growth and pubertal development are attributable to neuroendocrine dysfunction, which leads to a widespread disruption in hormone synthesis and metabolism, with far-reaching consequences $[4,7]$. A resulting suppression of gonadal function, coupled with alterations in the metabolism or peripheral actions of thyroid hormone and growth hormone are particularly detrimental to the integrity of the skeleton. In healthy children, $30-40 \%$ of adult bone mass is normally accrued during puberty under the anabolic influence of sex hormones, insulin-like growth factors and leptin $[48,49]$. A deficiency of these hormones under conditions of energy deprivation impairs bone acquisition. The outcome is low bone mass and fragile bone structure in adulthood [4, 49].

With nutritional rehabilitation, it is usually possible for previously undernourished children to gain weight, to undergo 'catch-up' growth and to complete pubertal maturation $[7,40]$. However, if nutritional insufficiency persists for many years, normal bone growth and mineral acquisition may be permanently impaired and genetically 'programmed' adult height may never be achieved. Under such circumstances, pubertal maturation is incomplete and reproductive capability is lost $[7,40]$. If nutritional insufficiency develops around the age of expected puberty, the pubertal growth spurt and subsequent maturation are delayed or inhibited $[7,40]$. The greater the severity of childhood energy deprivation and the longer this deprivation is endured, the poorer the prognosis for the prospective accomplishment of normal growth and maturation. Thus, when considering the nutritional requirements of child athletes, it is important to appreciate that their diets should encompass the specific nutritional demands of growth and maturation, in addition to the energy demands of physical training.

Ann Nestlé [Engl] 2006;64:63-76 
Nevertheless, it is noteworthy that some children who excel in certain sports (e.g. gymnastics and dance) are able to do so because of inherited small stature and late puberty. Such children often have small parents and siblings and present with constitutional growth delay (i.e. an inherent delay in the tempo of linear growth and weight gain); however, they may be adequately nourished and otherwise healthy $[7,40]$. At present, there is limited evidence to suggest that the retarded growth and maturation observed in various cohorts of child athletes are directly attributable to physical training per se. Rather, if the energy and specific nutrient demands of training are balanced through adequate nutrition, growth and maturation normally progress within normal boundaries $[3,50]$.

\section{Disordered Eating in Child Athletes}

Primary nutritional insufficiency in physically active children within developed societies is usually caused by deliberate, self-inflicted diet restriction rather than limited access to food. The term 'disordered eating' describes a pattern of unorthodox eating behavior that may fulfill a greater or lesser number of the formal diagnostic criteria of a clinical eating disorder such as anorexia or bulimia nervosa [51, 52]. Although disordered eating is normally psychogenic in origin, such behavior may be perpetuated by malnutrition and accompanying metabolic adaptations which impact detrimentally on cognitive functioning. Clinical eating disorders are more common in girls than in boys and usually develop after the onset of puberty, when a surge in the circulating levels of sex hormones effect rapid changes in body shape, fat/lean mass ratio and erratic emotional stability. Clinical eating disorders are characterized by ritualistic eating behaviors that are frequently associated with other obsessive-compulsive traits such as exercise dependence $[51,52]$.

Restrictive eating and 'excessive' but futile exercise are particularly harmful to adolescent growth, maturation and future health. There are reports that restrictive eating disorders are more widespread in certain athletic cohorts (e.g. distance runners, gymnasts, dancers and wrestlers) than in normally active individuals $[51,52]$. This finding could be explained by the pressure encountered or perceived by these young athletes to attain and maintain a lean physique, for the improvement of performance or aesthetic qualities. However, it is also possible that adolescents with an obsessive or otherwise vulnerable personality 'self-select' into such sports. For such adolescents, structured physical training may satisfy a need for routine and ritual, and sports clubs provide an environment in which leanness and repeated exercise are expected and accepted [51, 52].

Nutritional insufficiency in growing athletes may also arise from a simple lack of knowledge or misunderstanding of the importance of diet for the maintenance of performance and health, on behalf of the athlete, or their parents or coaches. Confusion and uncertainty surrounding the dietary requirements of the child athlete are comprehensible given the meager amount of published research on this topic. Children, particularly adolescents, may adopt dangerous dietary practices because they are subjected to misleading or unsubstantiated dietary advice through the media and popular magazines. It is for these reasons that child athletes should be carefully counseled by individuals with the appropriate dietetic expertise. Adolescents tend to be exceptionally sensitive to body image and may have distorted views of food and eating. Rather than to comprehend eating as an essential practice to drive normal metabolism, they may view food as an 'enemy' that will promote undesired weight gain $[24,51,52]$. Therefore, athletic children should be educated by significant others (e.g. coaches and parents) as regards the importance of food as vital fuel for optimal performance and health.

\section{General Sports Nutrition: The Special Importance of Nutrition for Athletes}

A well-balanced diet is essential for the maintenance of physical performance and the preservation of health in all individuals who engage in physical training, regardless of age. Food provides the chemical elements required for the production of energy by exercising skeletal muscle, the post-exercise replenishment of fuel stores and the regeneration of damaged muscle. Nutrition also modulates the circulating levels of anabolic and catabolic hormones that influence the metabolism of stored fuels during exercise and the capacity for post-exercise recovery $[21,53]$. The fundamental nutritional concern for individuals with high exercise energy expenditure is to ensure adequate energy intake. Such individuals have requirements for energy and specific nutrients that exceed those of their sedentary age- and sex-matched counterparts [8]. These requirements are governed by the extent to which exercise raises energy expenditure above the RMR, the duration of elevated energy expenditure, the total 'extra' energy cost of regular exercise, and the associated consumption and 
Table 1. The main factors that influence energy expenditure and fuel metabolism during exercise

Exercise intensity and duration, which influence the extent to which the muscles' aerobic and/or anaerobic energy systems are engaged and hence the 'choice' of metabolic fuel $[26,27]$

The frequency of training, which influences the capacity to recover fuel stores between repeated bouts of exercise $[17,20]$

The magnitude of fuel stores within the recruited muscle fibers before and after exercise, which comprise mainly glycogen and triglyceride $[17,20]$

Blood glucose concentration, which influences the release of hormones that maintain glycemic control, such as insulin, cortisol, catecholamines and growth hormone $[31,32]$

Habitual diet, particularly macronutrient intake, which also influences the magnitude of the muscles' fuel stores and blood glucose concentration $[17,20]$

Muscle fiber type, as based upon structural and biochemical properties, including the activity of enzymes that control the catabolism and storage of metabolic fuels $[12,17]$

Training 'status', as defined by the degree of metabolic adaptation to repeated, specific exercise, particularly within the recruited skeletal muscles $[12,17]$

The circulating levels of sex hormones, which are negligible in prepubertal children and increase with the onset of puberty $[23,36]$

possible depletion of specific fuels. Diet, energy balance and physical training also regulate body mass and body composition which influence sport performance capacity $[8,37]$.

Children and adults engaging in regular physical training are also likely to benefit from a higher intake of specific macro- and micronutrients, in excess of the recommendations for sedentary requirements. This is to assist energy production for exercise and tissue regeneration after exercise. The main factors that influence energy expenditure and fuel metabolism during exercise are illustrated in table 1.

The greater the mass of exercising muscle and the higher the power output of this muscle, the greater the rate of whole body energy expenditure. Higher exercise intensity and energy expenditure are also accompanied by a greater breakdown and utilization of glycogen relative to fat as muscle fuel, in both adults and children [26, 44]. Prolonged, high-intensity exercise in the absence of carbohydrate ingestion leads to a substantial reduction in muscle and liver glycogen stores $[16,17]$. Thus to prevent glycogen depletion and fatigue, all individuals who regularly undertake this type of exercise require an energy-balanced diet, of which at least $55 \%$ of energy is derived from carbohydrate $[8,24]$. Repeated exercise that engages the same muscle groups increases the risk of glycogen depletion within the specifically recruited muscle fibers. Therefore, the nutritional demands of physical training are strongly linked to the availability of carbohydrate, in the form of blood glucose and glycogen within liver and muscle $[16,17]$. Because of the abundance of stores of body fat relative to glycogen in healthy adults and children, the availability of lipids does not normally limit exercise performance. Thus, a high-fat diet has limited efficacy to muscle activity and physical performance, post-exercise recovery and training capacity in physically active individuals [20-22].

Differences in fuel metabolism during exercise between adults and children are mainly attributable to differences in physical size and to contrasting levels of circulating sex hormones $[22,23]$. These hormones influence fuel metabolism through their effects on body size and composition, the magnitude of the body's intra- and extramuscular fuel stores and the activity of various enzymes that control respiratory pathways within skeletal muscle. Discrepancies in the circulating levels of the various sex hormones may also underpin variations in fuel metabolism observed between men and women during exercise of a given relative intensity $[18,19]$.

\section{Post-Exercise Recovery}

Physical training provides the essential stimulus for adaptive changes in physiology and metabolism that may benefit or hinder performance and health $[53,54]$. Nutrition, energy balance and the circulating level of metabolic fuels play a central role in the process of post-exercise recovery. The latter describes the restoration of homeostasis after exercise and involves the repletion of energy stores alongside the synthesis of structural proteins and enzymes $[53,54]$. The effectiveness of recovery after exercise determines the nature of the metabolic and functional adaptations to a subsequent bout of exercise. Prolonged strenuous exercise, in particular, imposes considerable stress upon the major organ systems and elicits widespread catabolism. After exercise, this catabolism is reversed through combined rest and sound nutrition which enable a net anabolism. Anabolism promotes positive adaptations in the function of those tissues stressed by the exercise [53, 54]. An important feature of rest in the training program is that it is accompanied by a pattern of fuel metabolism 
Table 2. The key functions of nutrition for the promotion of favorable adaptations to physical training

Provision of metabolic fuel to skeletal muscle during exercise to balance the production and utilization of energy and hence to prevent fatigue $[13,16]$

Replenishment of fuel stores after exercise, especially muscle and liver glycogen, which are stored in limited amounts in the body when compared to fat $[13,16]$

Restoration of homeostasis after exercise, which includes fluid and electrolyte replacement and the normalization of blood glucose concentration $[21,53]$

Regeneration and repair of tissues of the musculoskeletal system damaged by exercise-induced micro-trauma to promote a 'supercompensation' (i.e. a superior anabolic response) $[21,53]$

within the skeletal muscle that contrasts with the pattern that occurs during exercise $[26,53]$. Rather than to catabolize large amounts of glycogen, the predominant energy substrate for resting skeletal muscle is fat. Low rates of glucose and glycogen oxidation promote glycogen resynthesis and hence the recovery of glycogen stores $[26,53]$.

Diet plays an important role in the adaptations to physical training. Failure to replace fuel stores after exercise, particularly muscle and liver glycogen, precludes a reversal of exercise-induced catabolism, limits the capacity for further exercise and may elicit unfavorable, maladaptive responses $[21,54]$. Sustained or repeated maladaptation may also lead to symptoms of 'over-training. The latter is characterized by disturbances in normal physiology, metabolism and mood, which impair performance, health and wellbeing $[54,55]$. Although the majority of research on the interaction of diet and physical training on performance and health has been conducted in adults rather than children, given the special requirements of optimal nutrition for childhood growth, it is logical to speculate that a child's susceptibility to ill-health when attempting to balance the competing demands of growth and physical training is greater than that of an adult. Thus, nutrition is a decisive factor in the capacity to profit from physical training because of its regulatory effects on the prevailing anabolic/catabolic balance.

The key functions of nutrition for the promotion of favorable adaptations to physical training are summarized in table 2 .

\section{Differences in the Nutritional Requirements of Child and Adult Athletes}

Although data are limited, it would appear that the primary physiological and metabolic features of exercise in children that differentiate their nutritional demands from those of adults are as follows:

(1) Increased lipid relative to carbohydrate oxidation during submaximal exercise of a given relative intensity which reduces the reliance upon glycogen as muscle fuel and may therefore reduce the importance of dietary carbohydrate as a muscle fuel $[35,44]$.

(2) Decreased mechanical efficiency during weightbearing activities, such as walking and running, which results in a higher energy expenditure per unit body mass and restricts the value of tables of energy expenditure for different activities derived from adult experimental data for use in children $[22,56]$.

(3) Decreased sweating and capacity to dissipate heat and regulate deep body temperature, which increases the requirement for fluid intake $[22,57]$.

These differences between children and adults diminish during adolescence alongside increases in body size and pubertal maturation $[2,23]$. To date, research has concluded that prepubertal children possess the metabolic and physiological attributes to perform effectively in prolonged (aerobic) exercise and activities that involve short bursts $(<10 \mathrm{~s})$ of intense activity that are driven by energy derived from the breakdown of ATP and creatine phosphate, such as jumping, throwing and short sprints. However, it would seem that prepubertal children are not well equipped physiologically to perform activities that are sustained by anaerobic glycolysis [22, 23]. Through adolescence, particularly in boys, there is enhancement of the capacity for anaerobic glycolysis that may be mediated through a development of the enzymatic control of this respiratory pathway and/or an increased capacity for glycogen storage $[22,23]$. During aerobic exercise, with increasing exercise intensity, children, like adults, metabolize relatively more endogenous carbohydrate stores (i.e. muscle glycogen and blood glucose) and proportionately less fat $[22,44]$. Interestingly however, children appear to oxidize relatively more exogenous carbohydrate ingested during exercise than do adults $[34,35]$. This may be because the capacity to catabolize endogenous glycogen for use as energy substrate by exercising muscle is less in children than in adults.

Children with a high physical activity level, particularly those undertaking strenuous physical training for competition in sport, have requirements for dietary en- 
ergy and specific nutrients that exceed those of sedentary or normally active children. Currently however, owing to limited research evidence, it is not precisely clear how the nutritional requirements of athletic children differ from those of their normally active counterparts. In adults, specific feeding strategies involving the ingestion of carbohydrate, with or without protein, before, during and after exercise, have been shown to promote physical performance and post-exercise recovery $[21,58]$. Carbohydrate feeding strategies augment glycogen storage before and after exercise, which assists the maintenance of an adequate blood glucose concentration and limits both glycogen and protein catabolism during exercise $[20,28]$. At present, the extent to which this type of diet manipulation enhances children's performance and post-exercise recovery is largely speculative. Caution has been expressed over the use of food supplements in both children [59] and adults [8].

\section{Fluid Balance and Thermoregulation}

Fluid and electrolyte balance are critical to the maintenance of homeostasis and physical performance. Muscle activity is largely inefficient, with over $75 \%$ of energy from metabolic fuels being transformed to heat rather than mechanical energy. This is especially true for children who have a lower mechanical efficiency than adults during walking and running [22, 56]. Excessive heat storage within the body results in hyperthermia and heatstroke. This is despite the operation of compensatory responses to an elevation in deep body temperature that include vasodilatation, which increases the convection of heat to the skin surface, and sweating, which increases heat loss through evaporation and cools the skin.

Failure to replace fluids and electrolytes (particularly sodium) lost through sweating leads to dehydration and electrolyte imbalances (e.g. hyponatremia) $[22,57]$. Moreover, children are at greater risk of heatstroke than adults because of a higher surface area to volume ratio, lower cardiac output, lower mechanical efficiency and underdeveloped compensatory responses to hyperthermia [22, 57]. The risk of heatstroke and dehydration is greatest when the environmental temperature exceeds $37^{\circ} \mathrm{C}$ and the level of humidity is too high to enable effective evaporative heat loss. Signs of heat illness include the excretion of scant quantities of dark, cola-colored urine, lethargy, nausea, headache and confusion.

Replacing lost fluid is necessary to sustain cardiac output, blood pressure and tissue perfusion. The latter assists the delivery of nutrients and oxygen and the removal of metabolic wastes. Because thirst is generally a poor indicator of fluid requirement in both adults and children, it is important to encourage drinking before, during and after exercise to prevent dehydration. The addition of small amounts of sodium chloride to water sensitizes the thirst mechanism through the maintenance of plasma osmolality and reduces the diuretic effect of plain water consumption $[8,22,57]$. For exercise bouts exceeding $1 \mathrm{~h}$, as has been shown in adults, the addition of carbohydrate $(8-10 \%)$ is likely to prevent hypoglycemia and may improve both performance and post-exercise recovery $[8$, $22,57]$.

\section{Practical Recommendations}

The primary objective of this article has been to justify the nutritional requirements of athletic children on the basis of the physiological and metabolic demands of survival, growth and physical activity. Nonetheless, there are obvious practical issues to consider when encouraging the consumption of an adequate diet in such children. These issues are the concern of parents, coaches and the children themselves, for whom scientific evidence should be translated into behavioral practice. The practical means of achieving a balanced diet has been addressed in detail previously [22, 24]. Informative advice may also be obtained from websites such as the Gatorade Sport Science Institute (www.gssiweb.com) and the United States Department of Agriculture Center for Nutrition Policy (www.usda.gov/ cnpp). The latter informs the pictorial 'Food Pyramid Guide' (www.mypyramid.gov), which is designed to provide an easily comprehensible means for the public to achieve a nutritious, well-balanced diet. This guide categorizes foods by their source and primary nutritional constituents (e.g. dairy, meat/fish, vegetables, fruits, cereals) and recommends quantities to be consumed by adults and children on the basis of portion sizes that provide an appropriate intake of all essential nutrients. For older adolescents (16-18 years), dietary recommendations made in the Joint Position Statement on Nutrition and Athletic Performance of ACSM, ADA and DC [8] are applicable and valuable.

As explained earlier, dietary energy and protein are especially pertinent to the maintenance of health and physical performance in growing athletes. The quantity of 'extra' energy required for growth varies in accordance with body size and maturity and is appropriately prescribed in relation to body mass in non-obese children. Energy and 
protein demands are greatest during the pubertal growth spurt and are higher in boys than girls because of a greater rate of increase in body size and body weight in the former $[1,24]$. During this phase of development, which typically occurs around the age of 8-10 years, normally active girls and boys usually require $65-75 \mathrm{kcal} / \mathrm{kg} /$ day, together with a protein intake of 1.1-1.2 g/day. By age 1213 years, energy and protein requirements have declined to $50-60 \mathrm{kcal} / \mathrm{kg} /$ day and $1.0-1.1 \mathrm{~g} /$ day, respectively, and by age $16-17$ years to $40-50 \mathrm{kcal} / \mathrm{kg} /$ day and $0.9-1.0 \mathrm{~g} /$ day $[22,24]$. The higher of these energy and protein demands generally apply to boys owing to their larger size. The energy cost of physical training should be accounted for and added to these requirements.

In healthy children, the high energy demands associated with physical training are usually achieved through a natural increase in appetite, and it is important that this energy is derived from a nutritious, well-balanced diet. Adherence to the Food Pyramid Guide provides energy in approximate macronutrient proportions of $55-60 \%$ carbohydrate, $12-15 \%$ protein and $25-30 \%$ fat. At present there is a paucity of data to qualify the effects of physical training on protein requirements in growing athletes. However, it is feasible that, as shown in mature athletes [21], child athletes require $50-80 \%$ more protein than their normally active counterparts to prevent negative nitrogen balance. Nevertheless, it is likely that ensuring the maintenance of energy balance and the aforementioned proportions of dietary macronutrients will adequately cover the growing athlete's requirements for protein and additional carbohydrate. The latter is 'protein sparing' in that it limits the use of protein as a substrate for both ATP synthesis and gluconeogenesis $[8,24]$. A risk of protein deficiency is possible in children who follow a vegetarian diet, particularly if they exclude fish and dairy produce which, like meat, contain all essential amino acids. For vegetarian child athletes, a prudent choice of mixed protein foods (e.g. pulses, cereals and nuts) is essential to ensure a balanced and adequate intake of all essential amino acids.

Growth and regular exercise also demand the consumption of foods that provide sufficient quantities of micronutrients to control essential metabolic pathways involved in the synthesis of ATP and new tissue. During growth, iron and calcium are required in elevated amounts for the synthesis of hemoglobin and bone mineral, respectively. Of importance, nutritional studies in normally active children within the USA [24] and UK [60] have demonstrated deficiencies of these particular minerals, and also the vitamins $\mathrm{C}, \mathrm{E}$ and $\mathrm{B}_{6}$. Although these deficiencies are largely attributable to dietary deficiency, a growth-re- lated increase in demand for these micronutrients is also a contributory factor. Physically active children should be encouraged to drink regularly $(100-150 \mathrm{ml} / \mathrm{h})$ throughout the day and, depending upon body size, to consume an extra $200-400 \mathrm{ml}$ of water within the $2 \mathrm{~h}$ preceding training, $150-200 \mathrm{ml}$ every 15-20 min during exercise and 400-600 ml immediately after training [57]. The maintenance of fluid balance and hydration state in children may be enhanced through the provision of highly palatable (e.g. flavored) drinks.

\section{Conclusions}

The primary goals of childhood are optimal growth and maturation, both of which demand positive energy and nitrogen balance. Children engaging in strenuous sports should therefore consume a diet that adequately satisfies the nutritional demands of both their physical training and normal growth. The dietary requirements of physically active children alter with increasing body size and pubertal progression. The maturation state influences the physical performance capacity and dietary needs through its effects on the metabolic and physiological responses to exercise. Prepubertal children are physiologically well equipped to perform prolonged bouts of aerobic activity, but their anaerobic performance is limited. Like physically active adults, active children have greater dietary demands for energy, carbohydrate, protein and water than their normally active or sedentary counterparts. 


\section{References}

1 Malina RM: Physical growth and biological maturation of young athletes. Exerc Sports Sci Rev 1994;22:389-433.

2 Naughton G, Farpur-Lambert NJ, Carlson J, Bradney M, Van Praagh E: Physiological issues surrounding the performance of adolescent athletes. Sports Med 2000;30:309-325.

3 Roemmich JN, Richmond EJ, Rogel AD: Consequences of sport training during puberty. J Endocrinol Invest 2001;24:708-715.

-4 Zanker CL Cooke CB: Energy balance, endocrine function and bone health. Med Sci Sports Exerc 2004;36:1372-1381.

5 Soyka LA, Grinspoon S, Levitsky LL, Herzog DB, Klibanski A: The effects of anorexia nervosa on bone metabolism in female adolescents. J Clin Endocrinol Metab 1999;84:44894496.

6 Warren MP: The effects of exercise on pubertal progression and reproductive function in girls. J Clin Endocrinol Metab 1980;51:11501157.

7 Rogol AD, Clark PA, Roemmich JN: Growth and pubertal development in children and adolescents: effects of diet and physical activity. Am J Clin Nutr 2000;72(suppl):521S-528S.

8 Manore MM, Barr SI, Butterfield GE: Nutrition and Athletic Performance. Joint Position Statement of ACSM/ADA/DC. Med Sci Sports Exerc 2000;32:2130-2145.

9 Medical Research Council Ethics Guide. Medical Research Involving Children. London, Medical Research Council, 2004.

10 McIntosh N, Bates P, Brykczynska G, Dunstan G, Goldman A, Harvey D, Larcher V, McCrae D, McKinnon A, Patton M, Saunders J, Shelley P: Guidelines for the ethical conduct of medical research involving children. Royal College of Paediatrics and Child Health: Ethics Advisory Committee. Arch Dis Child 2000;82: 177-182.

11 International Ethical Guidelines for Biomedical Research Involving Human Subjects. Geneva, Council for International Organizations of Medical Sciences, 2002.

$\checkmark 12$ De Feo P, Di Loreto C, Lucidi P, Murdolo G, Parlanti N, De Cicco A, Piccioni F, Santeusanio F: Metabolic response to exercise. J Endocrinol Invest 2003;26:851-864.

13 Hagerman FC: Energy metabolism and fuel utilization. Med Sci Sports Exerc1992;24: S309-S314.

14 Ainsworth BE, Haskell WL, Whitt MC, Irwin ML, Schwartz, AM, Strath SJ, O'Brien WL, Bassett DR Jr, Schmitz KH, Emplaincourt PO, Jacobs DR Jr, Leon AS: Compendium of physical activities: an update of activity codes and MET intensities. Med Sci Sports Exerc 2000; 32:S498-S504.

15 Hendricks KM, Duggan C, Walker WA: Manual of Pediatric Nutrition, ed 3. Hamilton, Decker, 2000, p 142.
16 Brooks GA: Mammalian fuel utilization during sustained exercise. Comp Biochem Physiol Biochem Mol Biol 1998;120:89-107.

17 Coyle EF: Substrate utilization during exercise in active people. Am J Clin Nutr 1995;61(suppl):S968-S979.

18 Tarnopolsky LJ, MacDougall JD, Atkinson SA, Tarnopolsky MA, Sutton JR: Gender differences in substrate for endurance exercise. Appl Physiol 1990;68:302-308.

19 Horton TJ, Pagliassotti MJ, Hobbs K, O’Hill J: Fuel metabolism in men and women during and after long-duration exercise. J Appl Physiol 1998;85:1823-1832.

20 Spriet LL, Peters SJ: Influence of diet on the metabolic responses to exercise. Proc Nutr Soc 1998;57:25-33.

21 Kreider RB, Miriel V, Bertun E: Amino acid supplementation and exercise performance. Analysis of the proposed ergogenic value. Sports Med 1993;16:190-206.

22 Bar-Or O: Nutritional considerations for the child athlete. Can J Appl Physiol 2001;26(suppl):S186-S191.

23 Boisseau N, Delamarche P: Metabolic and hormonal responses to exercise in children and adolescents: Sports Med 2000;30:405-422.

24 Steen SN: Nutrition for the school age athlete; in Berning JR, Steen SN (eds): Nutrition for Sport and Exercise. Gaithersburg, Aspen, 1998, pp 217-245.

25 Green HJ: Mechanisms of muscle fatigue in intense exercise. J Sports Sci 1997;15:247256.

26 Romijn JA, Coyle EF, Sidossis LS, Gastaldelli A, Hoeowitz JF, Endert E, Wolfe RR: Regulation of endogenous fat and carbohydrate metabolism in relation to exercise intensity and duration. Am J Physiol 1993;265:E380-E391.

27 Brooks GA, Mercier J: Balance of carbohydrate and lipid utilization during exercise: the 'crossover' concept. J Appl Physiol 1994;76: 2253-2261.

28 Wagenmakers AJM, Beckers EJ, Brouns F Kuipers H, Soeters PB, Van Der Vusse GJ, Saris WHM: Carbohydrate supplementation, glycogen depletion and amino acid metabolism during exercise. Am J Physiol 1991;260 E883-E890.

29 Kasperek GJ, Snider RD: Effect of exercise intensity and starvation on activation of branched-chain keto-acid dehydrogenase by exercise. Am J Physiol 1987;252:E33-E37.

30 Zanker CL, Swaine IL, Castell L, Newsholme EA: Responses of plasma glutamine, free tryptophan and branched-chain amino acids to prolonged exercise after a regime designed to reduce muscle glycogen. Eur J Appl Phys 1997; 75:543-548

31 Galbo H: Exercise physiology: humoral function. Sport Sci Rev 1992;1:65-93.

32 Viru A: Stability and variability in hormonal responses to prolonged exercise. Int J Sports Med 1992;13:230-234.
33 Martinez LR, Haymes EM: Substrate utilization during treadmill running in prepubertal girls and women. Med Sci Sports Exerc 1992; 24:975-983.

34 Riddell MC, Bar-Or O, Wilk B, Parolin ML, Heigenhauser GJ: Substrate utilization during exercise with glucose and glucose plus fructose ingestion in boys ages 10-14 yr. J Appl Physiol 2001;90:903-911.

35 Timmons BW, Bar-Or O, Riddell MC: Oxidation rate of exogenous carbohydrate during exercise is higher in boys than in men. J Appl Physiol 2002;94:278-284.

36 Berg A, Keul J: Biochemical changes during exercise in children; in Malina RM (ed): Young Athletes: A Biological, Psychological and Educational Perspective. Champaign, Human Kinetics, 1988, pp 61-67.

37 Astrand PO, Rodahl K: Textbook of Work Physiology: Physiological Bases of Exercise. New York, McGraw-Hill, 1986, pp 295-353.

38 Biddle S, Sallis J, Cavill N: Young and Active? Young People and Health-Enhancing Physical Activity: Evidence and Implications. London, Health Education Authority, 1998.

39 Zanker CL, Gannon L, Cooke CB, Gee KL, Oldroyd B, Truscott JG: Differences in bone density, body composition, physical activity and diet between child gymnasts and untrained children aged $7-8$ years. J Bone Miner Res 2003; 18:1043-1050.

40 Caine D, Lewis R, O’Connor P, Howe W, Bass $\mathrm{S}$ : Does gymnastics training inhibit growth of females? Clin J Sport Med 2001;11:260-270.

41 Armstrong N, Welsman JR: Developmental aspects of aerobic fitness in children and adolescents; in Holloszy JO (ed): Exercise and Sports Science Reviews. Baltimore, Williams \& Wilkins, 1994, pp 435-476.

42 Saltin B: Oxygen transport by the circulatory system during exercise in man; in Keul J (ed): Limiting Factors of Physiological Performance. Stuttgart, Thieme, 1973, pp 235-252.

43 Pate RR, Kriska A: Physiological basis of the sex difference in cardiorespiratory endurance. Sports Med 1984;1:87-90.

44 Foricher JM, Ville N, Gratas-Delamarche A, Delamarche P: Effects of submaximal intensity cycle ergometry for one hour on substrate utilization in trained prepubertal boys versus trained adults. J Sports Med Phys Fitness 2003;43:36-43.

45 Golden K, Waterlow JC, Picou D: The relationship between dietary intake, weight change, nitrogen balance and protein turnover in man Am J Clin Nutr 1977;30:1345-1348.

46 Matkovic V, Ilich J, Skugor M, Badenhop NE, Goel P, Clairmont A, Klisovic D, Nahhos RW, Landoll JD: Leptin is inversely related to age at menarche in human females. J Clin Endocrinol Metab 1997;82:3239-3245. 
-47 Welt CK, Chan JL, Bullen J, Murphy R, Smith P, DePaoli AM, Karalis A, Mantzoros CS: Recombinant human leptin in women with hypothalamic amenorrhea. N Engl J Med 2004; 351:987-997.

48 Bonjour J, Theintz G, Buchs B, Slosman D, Rizzoli R: Critical years and stages of puberty for spinal and femoral bone mass accumulation during adolescence. J Clin Endocrinol Metab 1991;73:555-563.

$\checkmark 49$ Weaver CM: Adolescence: the period of dramatic bone growth. Endocrine 2002;17:4348.

50 Baxter-Jones ADG, Helms PJ: Effects of training at a young age: review of the training of young athletes study. Pediatr Exerc Sci 1996: 8:310-327.

51 Wilson GT, Eldridge KL: Pathology and development of eating disorders: implications for athletes; in Brownell KD, Rodin J, Wilmore JH (eds): Eating, Body Weight and Performance in Athletes. Disorders of Modern Society. Philadelphia, Lea \& Febiger, 1992, pp 115 127.
52 Sundgot-Borgen J: Risk and trigger factors for the development of eating disorders in female elite athletes. Med Sci Sports Exerc 1994;26: 414-419.

53 Viru A: Post-exercise recovery period: carbohydrate and protein metabolism. Scand J Med Sci Sports 1996;6:2-14.

54 Kuipers H, Keizer HA: Overtraining in elite athletes: a review and directions for the future. Sports Med 1988;6:79-92.

55 Fry RW, Morton AR, Keast D: Overtraining in athletes: an update. Sports Med 1991;12:3265.

56 Frost G, Dowling J, Dyson K, Bar-Or O: Cocontraction in three age groups of children during treadmill locomotion. J Electromyog Kinesiol 1997;7:179-186.
57 Rivera-Brown AM, Gutierrez R, Gutierrez JC, Frontera WR, Bar-Or O: Drink composition, voluntary drinking and fluid balance in exercising, trained, heat-acclimatized boys. J Appl Physiol 1999;86:78-84.

58 Zawadski KM, Yaspelkis BB, Ivy JL: Carbohydrate-protein complex increases the rate of muscle glycogen storage after exercise. J Appl Physiol 1992;72:1854-1859.

59 Johnson WA, Landry GL: Nutritional supplements: fact vs. fiction. Adolesc Med 1998;9: 501-513.

60 Gregory J, Lowe S, Bates CJ, Prentice A, Jackson LV, Smithers G, Wenlock R, Farron M National Diet and Nutrition Survey: Young People Aged 4 to 18 Years. Vol 1: Report of the Diet and Nutrition Survey. London, TSO, 2000 . 